\title{
Assessing the Recent Impact Flux in the Inner Solar System: 1 Ga to Present
}

White Paper for the Planetary Decadal Survey, 2020-2030

R. R. Ghent, Planetary Science Institute, Tucson AZ 85719, rghent@psi.edu, 619-481-4321

N. E. B. Zellner, Department of Physics, Albion College, Albion, MI 49224, nzellner@albion.edu, 517-629-0465

\section{Co-authors:}

E.S. Costello, University of Hawaii at Manoa, Honolulu, HI 96822

I. Daubar, Brown University, Providence, RI 02912

C. I. Fassett, NASA Marshall Space Flight Center, Huntsville, AL 35801

M. Kirchoff, S. Marchi, and S. J. Robbins, Southwest Research Institute, Boulder, CO 80302

J.-P. Williams, University of California, Los Angeles, CA 90024

\section{Synopsis}

A major goal of current planetary science and exploration efforts is to quantify the recent flux of impactors affecting the terrestrial planets. This is an important undertaking because impact craters represent the only means of determining the absolute ages of features, and therefore, the rates of geological processes, on solar system bodies from which we have no samples. The connection between impact crater populations on all inner solar system bodies and the absolute time scale depends on analyzing the geochemistry and absolute ages of samples from Earth's Moon, the only body from which samples have been obtained. However, large gaps in the age spectrum of existing returned lunar samples hamper our ability to investigate processes occurring in certain time periods. For example, multiple lines of evidence suggest that the impact rate on the Moon has changed over the past 1 billion years. As the pace of data acquisition from the inner planets increases, though, we have an unprecedented opportunity to investigate impact events that may be correlated across planetary bodies, including Earth. An integrated approach that combines sample analyses with orbital observations and dynamical models is required. In the next decade we should answer questions such as: What are the quantitative constraints on the Earth-Moon impact flux during the last 1 billion years? Do similar impact ages assigned to some craters on the Moon, Mercury, Mars, and Earth mean they were formed at the same time? Are the craters associated in time with other geological or biological events correlated or coincident? To that end, we recommend:

- Sample collection from well-documented and thoughtfully chosen landing sites on the Moon;

- Continued support and increased development of in situ radiometric dating and seismic detection techniques;

- Continued support and development of Earth-based sample analysis laboratories;

- Continued work to determine the flux of extraterrestrial material to Earth at different times;

- Continued support to observe and catalog the population of near-Earth objects;

- Increased efforts to monitor lunar impact flashes from Earth and to obtain new seismic observations of current lunar impacts; and

- Supporting a diverse and inclusive workforce to achieve these objectives.

\section{Signatories}

See the list of community supporters at RecentImpactFlux 


\section{Introduction and Significance of Establishing the Recent Impact Flux}

The evolution of the inner solar system bodies is intimately tied to the history of bombardment. Impacts shape every aspect of airless bodies, from the surfaces of regolith grains to crustal structures. On surfaces with significant atmospheres, like Venus or Earth, the population of larger impact craters also reflects the history of resurfacing. The impact rate - and variations in this rate over time and space - are thus both of fundamental importance for understanding the histories of individual bodies and of the solar system as a whole. The craters produced by impacts provide an indelible record of events affecting terrestrial bodies and can be used to unravel their geological histories. They also provide information about the dynamic conditions within the solar system, expressed as variations in the characteristics (e.g., size-frequency distributions, SFDs) of the impactor population. The lunar impact flux record has been studied since the late 19th century (e.g., l) and more intensively for the past half-century, since the Moon's craters were recognized as impact features (2) and impact samples returned by the Luna and Apollo missions have been analyzed. Yet only now, with the benefit of more than a decade of intensive new spacecraft observations and focused sample investigations, can we begin to ask and answer critical questions that will lead to a deeper understanding of the fundamental processes at work.

The Moon's impact history is of especial importance to understanding geological processes on other solar system bodies, because the lunar impact record - and its calibration, through returned samples, to an absolute time scale - forms the basis for all planetary geological timescales. The Moon's geologic history is commonly divided into five major epochs, based in part on its impact record: Pre-Nectarian (the early history until the formation of Nectaris); Nectarian (from the formation of Nectaris to the formation of Imbrium, 3.9 Gyr ago); Imbrian (from the formation of Imbrium until $~ 3.2$ Gyr ago); Eratosthenian (its "middle ages," $\sim 3.2$ to $~ 1$ Gyr ago); and Copernican (its "recent" history, $\sim 1$ Gyr ago to the present). Major outstanding questions are associated with each of these periods. Those associated with the early and middle histories of the Moon are addressed elsewhere (e.g., 3-10). In this paper, we focus on the recent period, from $\sim 1$ $\mathrm{Ga}$ to the present. Events during this period are particularly important because of their influence on the geological and biological evolution of the inner solar system bodies. For instance, on Earth, this period spans both the Cambrian explosion, during which terrestrial life rapidly evolved increasingly complex life forms, and the period of literally life-shattering effects from the $\sim 65 \mathrm{Ma}$ Chicxulub impact (e.g., 11); it is possible that other, earlier events may have had equally profound effects on the directions of biological evolution on Earth. In another example, the past 1 billion years may have seen one or more large impacts that delivered water to the poles of Mercury, fundamentally altering its surface volatile budget $(12,13)$. Despite similar water delivery and removal processes operating on both Mercury and the Moon, the Moon does not show the same unambiguous signature of extensive and pure ice deposits at its poles. This source of this difference is rooted in bombardment: at the large scale (responsible for rapid volatile delivery e.g., 13); the very small scale (responsible for the delivery, disruption, and/or sequestration of water ice by micrometeorites and impact gardening; e.g., 12, 14); or both.

These examples illustrate that we currently have an unprecedented opportunity to investigate impact events that may be correlated across bodies. An integrated approach is required to gain a clearer understanding of the inner solar system and its evolution as a whole. In the next decade we should address the questions: How did the impact flux vary over the past billion years, and how do we know? Do similar impact ages assigned to some craters on the Moon, Mercury, Mars, and 
Earth mean they were formed at the same time, and if so, what do they tell us about the impactor population?
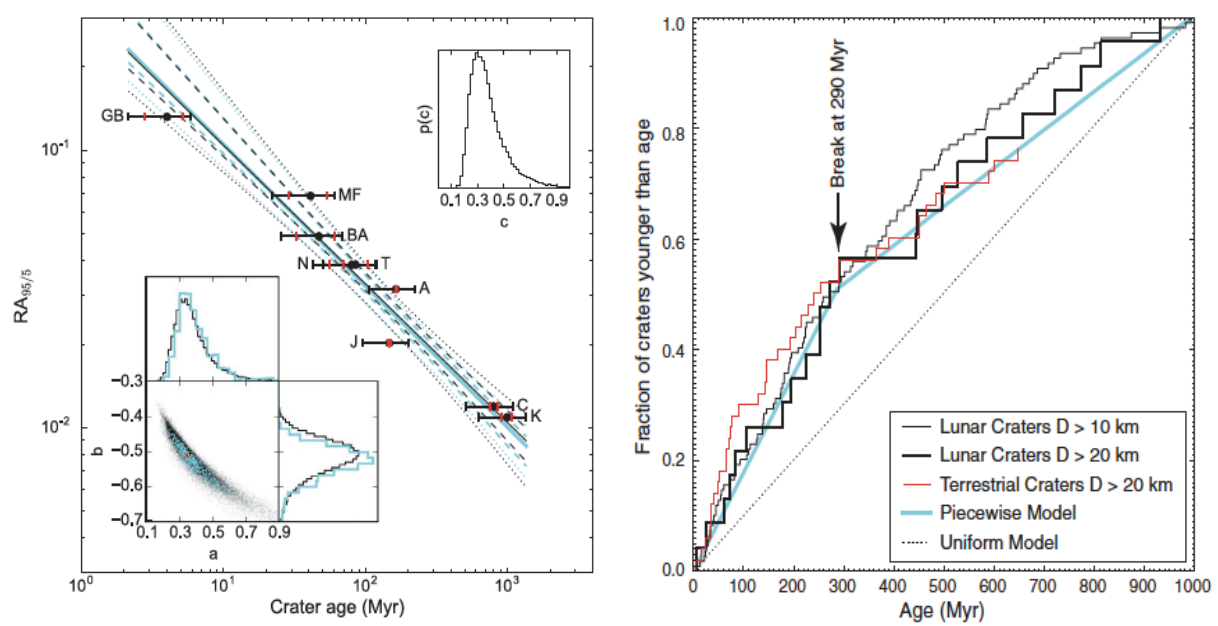

Figure 1: Left: Evolution of lunar crater ejecta properties with age provide method for dating lunar craters. Right: Resulting age spectrum shows nonuniform cratering rate. Terrestrial craters show the same signal (17).

Community Consensus: The importance of the bombardment history of the Moon, and more generally, the need for geochronological information that can link that history to solar system evolution, is reflected in a number of community consensus documents. For instance, the National Research Council's 2007 SCEM report (15) lists the need for geological chronological constraints in several of the fundamental concepts: Concept 1: The bombardment history of the inner Solar System is uniquely revealed on the Moon, with the goal to Establish a precise absolute chronology (1c) and assess the recent impact flux (1d); $\underline{6}$ : The Moon is an accessible laboratory for studying the impact process on planetary scales; $\underline{7}$ : The Moon is a natural laboratory for regolith processes and weathering on anhydrous airless bodies. Additionally, the 2016 Lunar Exploration Roadmap (16) lists goals, objectives, and investigations that include the following: Goal: Understand the formation, evolution, and current state of the Moon, with the objective to determine the stratigraphy, structure, and geological history of the Moon; Goal: Use the Moon as a "witness plate" for solar system evolution, with the objective to Understand the impact history of the inner Solar System as recorded on the Moon, by determin[ing] the impact flux throughout the postbasin-forming epoch. Finally, the ideas and recommendations discussed here are connected to those presented in the white papers entitled, "Exploring the Bombardment History of the Moon" presented by Bottke, "Geochronology as a Framework for Inner Solar System History and Evolution" presented by Cohen et al., and "Investigating Impact Processes at all Scales: The Moon as a Laboratory" by Costello and Potter et al.

\section{A Solar System View}

Modern approaches to planetary science consider the solar system as just that: a system. The impact flux in the inner solar system, and variations in that flux, have implications for the evolution of the entire system. We have begun to understand the need to study events with temporal similarities on multiple bodies as possible signatures of larger, system-wide evolutionary processes. Examples from the literature of temporally overlapping events occurring in the past 1 Gyr include the following.

- Analysis of the age spectrum of large craters with ages $<650 \mathrm{Ma}$ on both the Moon and Earth reveal evidence for a non-uniform cratering rate on both bodies, with a step-wise factor of 23 increase at $290 \mathrm{Ma}(17)$; Fig. 1 right). 
- Ages of fossil chondrite meteorites (e.g., 18) found in lower Ordovician (480 Ma) sediments and ages of several terrestrial impact craters (19) provide evidence for an enhanced impact flux that may be dynamically linked to the break-up of the L-chrondrite asteroid parent body (18-22). The debris from these impacts may have initiated the mid-Ordovician ice age (23). Further studies of fossil meteorites have expanded the temporal window beyond the lower Ordovician and constrain fall rates of various meteorite types over the last billion years (e.g. pre-Ordovician breakup: 24; post-Ordovician breakup: 25; Late Silurian: 26; Devonian Frasnian-Famennian boundary: 27; Jurassic: 28; Early Cretaceous: 29).

- Global melting of Snowball Earths (660-710 Ma, 645-655 Ma) may have been influenced by water vapor lofted into the terrestrial atmosphere during coincident periods of enhanced impact flux (30). Interestingly, this melting and associated erosion may be responsible for the observed lack of large terrestrial craters and spatially associated kimberlite diatremes on cratonic terrains, expressed as a sharp cutoff in the number of both features at $650 \mathrm{Ma}$ relative to later times $(17,31)$.

- Evidence for 800-Ma impacts has been supported by formation ages of lunar impact glasses (32; Fig. 3) and ages of impact melts in H- and L-chondrites $(33,34)$ and samples of Chelyabinsk (35). Formation of the Flora family of asteroids $(36,37)$ may be responsible.

- In addition to the record of terrestrial craters, there are $\sim 20$ impact spherule layers or tektite strewn fields younger than $2.0 \mathrm{Ga}$ that are not associated with known craters (38).

Interpretations that tie these events together within each proposed timeframe are limited by small data sets, lack of well-characterized terrestrial geologic evidence, and/or large uncertainties in derived or estimated sample or crater ages. More data are needed.

\section{Current Assessments of the Moon's Impact Flux}

Crater-count Ages. Our current understanding of the lunar impact flux from $1 \mathrm{Ga}$ to the present is based on interpretation of crater formation ages and on the ages of Luna and Apollo impact

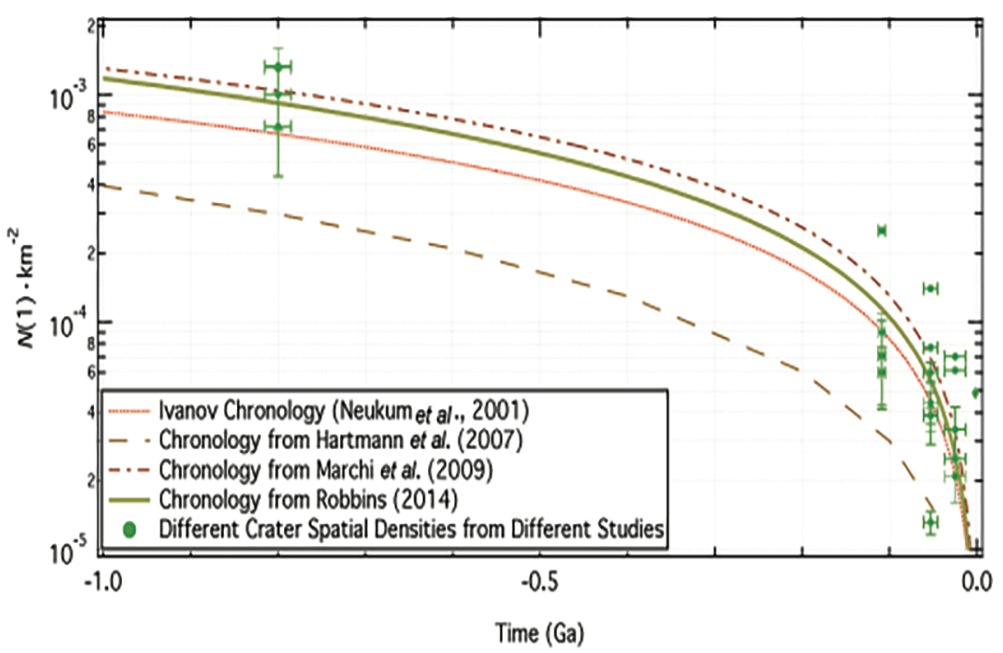

Figure 2: Plot of data (green points) and some lunar chronology models (lines) for the past 1 Ga for the Moon. Horizontal axis: radiometric ages of Copernicus, Tycho, North Ray, South Ray, and Cone craters based on Apollo 12 and 16 samples; vertical axis: crater spatial densities. samples from presumed known locations. The size-frequency distribution of lunar impact craters $<1 \mathrm{~km}$ to $200 \mathrm{~km}$ in diameter describes a "production function" that is canonically taken to reflect the flux of crater-creating impactors over the history of the Moon (e.g., 39; Fig. 2). This production function is calibrated to an absolute time scale via radiometric ages of Apollo and Luna samples collected at volcanic and impact sites. The combination of the resulting chronology function and counts of small craters superimposed on individual features or geological terrains gives absolute model ages (AMAs), in years, for those features (e.g., 9, 10, 40- 43). 
The canonical empirical chronology functions are anchored by the ages of a small number of returned lunar samples, which are all either $>3 \mathrm{Gyr}$ or $<800 \mathrm{Myr}$ in age; no samples exist with ages between $800 \mathrm{Myr}$ and $3.2 \mathrm{Gyr}$ (Fig. 2). This large gap in the sample record represents a major source of uncertainty for defining the overall lunar chronology function, and the lack of data makes it challenging to accurately assign ages to lunar features $<1$ Gyr. Additional complications include: (1) systematic uncertainties in the absolute time scale calibration that can lead to age uncertainties that are both large and difficult to quantify (Fig. 2); (2) crater counts that inherently average the terrains on which the counts are performed; (3) unrecognized secondary craters that can inflate crater counts and distort SFD curves; and (4) counts on crater floors that yield systematically younger ages than ejecta counts (44-46; and 47 showed this on Mars as well). The latter two effects lead to a large spread in crater spatial densities calculated by different researchers. These uncertainties allow for different proposed chronology functions, all of which satisfy the available constraints (e.g., 5, 8). These have important implications for recent events, and current data are insufficient to discriminate among them.

Lunar Samples. A complete understanding of the lunar impact flux - and, ultimately, the flux in the rest of the solar system-requires a fully calibrated absolute chronology that reliably determines the ages of samples with known provenance. At present, absolute ages of a handful of young craters - Copernicus, Tycho, North and South Ray, and Cone craters - have been determined using radiometric or cosmic ray exposure techniques on returned samples. However, the ages of Copernicus and Tycho have been called into question (e.g., 48, 49) because their absolute ages were indirectly determined (e.g., 50). Thus, the absolute ages of these craters may or may not be represented in the sample collection, rendering the present-day absolute time scale calibration uncertain. Other young impact samples have been identified in the lunar sample collection; however, they have no known provenance. For example, there is a preponderance of samples (e.g., impact glasses, impact melts in Apollo samples, and meteorites) with impact formation or melt ages $\sim 800 \mathrm{Ma}, \sim 660$ $\mathrm{Ma}$, and $\sim 80 \mathrm{Ma}$, and these multiple samples with

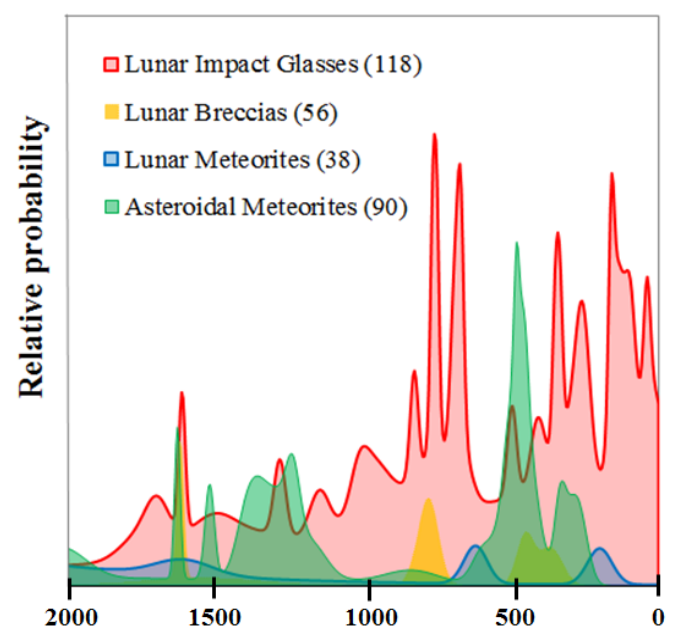

Figure 3: Recent impact episodes, as represented by a variety of solar system samples $\left({ }^{40} \mathrm{Ar}{ }^{\beta 9} \mathrm{Ar}\right.$ ages).

Modified from 52, refs therein. similar young ages could be interpreted to represent an enhanced impact flux or variations in the impact flux over the past 1 Gyr (Fig. 3). More generally, other lunar samples such as impactites and impact glasses identified in the Apollo sample collection, impact melts in meteorites from the Moon, Mars, and asteroids, and terrestrial tektites and impact spherules represent a rich catalog of potential relative and/or absolute age information via radiometric dating systems. Finally, the composition of samples varies with age: a study of Apollo 16 ancient regolith breccias reported that the Moon experienced impacts by a diverse group of impactors between 1.7 and 0.65 and $<0.5 \mathrm{Ga}$, in contrast to earlier times (51). To capitalize on this potential and to retire the uncertainties associated with crater counting and absolute chronologies, we need new samples from the Moon with 1) known provenance; and 2) 
sufficient variety in age, composition, and source terrain to allow us to constrain the range of variability in the relationship between crater counts and sample absolute ages.

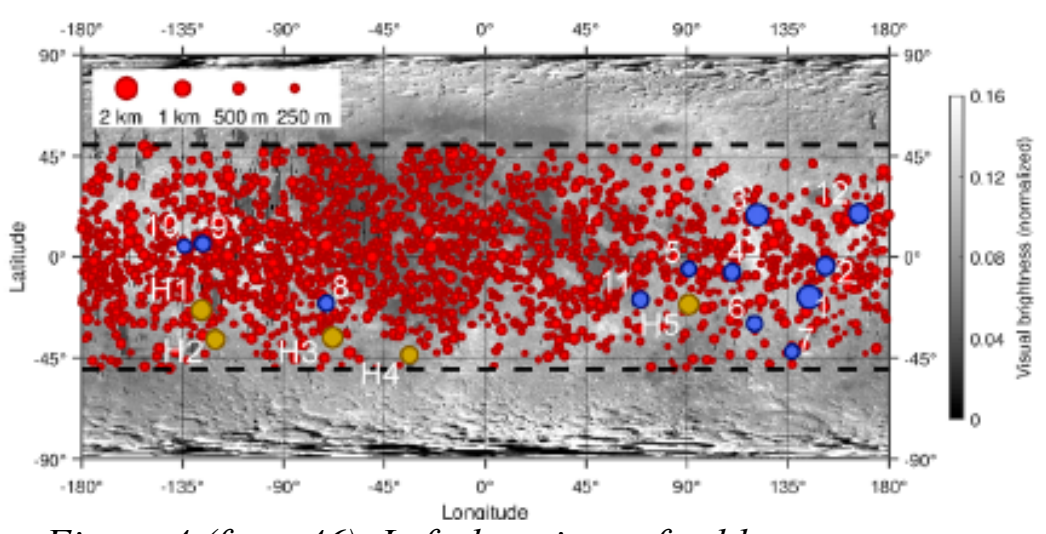

Figure 4 (from 46). Left: locations of cold spot craters on Diviner map of visible surface brightness. Blue markers: $D>800-m$ craters (labeled 1-12). Right: Cumulative SFDs of cold spot craters for latitudes $>20^{\circ} \mathrm{N}$ with isochrons from (39).

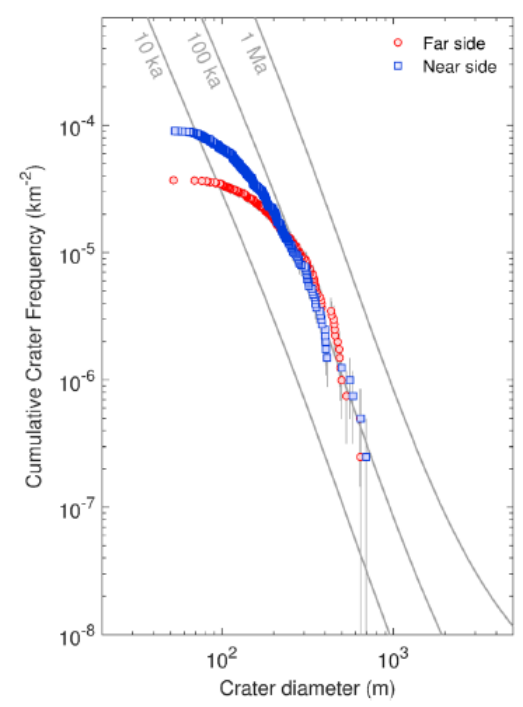

Physical Properties of Crater Ejecta: In addition to crater counts performed on large craters' ejecta and/or floors, the physical state of large craters is correlated with their age. Various authors have proposed relationships between crater age and morphological characteristics such as the presence or absence of rays (e.g., 3), or the maturation state of the ejecta (53). Using LRO Diviner thermal radiometer data (54), an inverse relationship between the rockiness of young craters' ejecta and crater age was documented (55; Fig. 1). This provides a new way to estimate absolute ages for otherwise undated craters younger than $1 \mathrm{Ga}$, and results in an age spectrum for lunar craters that allows analysis of the impact flux over that period. Following from this result, strong evidence for a two- to three-fold increase in the flux of bodies that produce $10 \mathrm{~km}$ and larger craters at $\sim 290$ Myr ago has emerged (17). The terrestrial population shows evidence for the same increase (Fig. 1 right). LRO Diviner data have also revealed the existence of $>2000$ "cold spots" associated with recently-formed small craters (56). These small craters show anomalously cold nighttime temperatures to distances of $\sim 10$ to $>100$ crater radii. Their formation mechanism is unknown, but the SFD of source craters, and crater counts on the ejecta of the largest cold spot craters, indicate that cold spots degrade rapidly, surviving no longer than $\sim 1 \operatorname{Myr}(46,57)$. The three largest fresh impacts $(D>40 \mathrm{~m})$ identified by LROC temporal pair imaging $(60)$ have generated cold spots and it is likely that cold spots are common to all recent impacts. They thus provide a means of identifying the population of craters that have formed over the last $\sim 100$ ka to $\sim 1 \mathrm{Ma}$, likely by a population of objects with relatively low mean encounter velocities $(\sim 8.4 \mathrm{~km} / \mathrm{s} ; 46)$.

Direct Observation of Recent Impacts: The most recent impacts in lunar history have been captured with modern high-resolution orbital imaging, including LROC Narrow Angle Camera (NAC) temporal image (before and after) pairs, and Earth-based observations of lunar impact "flashes," (e.g., 58; see also the white paper entitled, "Assessing the Present Day Impact Flux to the Lunar Surface via Impact Flash Monitoring and its Implications for Sustained Lunar Exploration" by Cahill and Speyerer et al.). Newly-formed craters and so-called "splotches," or image reflectance anomalies that appear in temporal pairs and thought to result from small primary 
or secondary impacts, constrain the current flux of meter- and smaller scale impacting objects to be significantly higher than predicted by the standard production and chronology functions (5961). This flux predicts a processing rate for the upper $2 \mathrm{~cm}$ of lunar regolith that is 100 times faster than previously thought (60). Continued observations over longer baselines are needed to extend this constraint to larger craters, and thus adjust standard chronologies in a way that is broadly applicable to older surfaces and other bodies, such as Mars (e.g., 62). More work remains to be done to explain the steeper SFD of current impacts, connecting these observations to dynamics of orbiting bodies and impacting populations, and understanding the effects of secondary cratering on the observed SFD.

The modern flux can be elucidated from studies of micrometeorites (63) and was also directly observed by the Apollo Passive Seismic Experiments, which recorded on average 200 impacts/year over the lifetime of the array (e.g., 64-66). Source modeling of the seismic signature of the impact process suggests the larger recorded impacts may have produced craters $73 \mathrm{~m}$ in diameter (67) and production functions for the past 40 years of activity suggest $>1000$ impacts producing craters with diameters of $10 \mathrm{~m}$ or greater across the entirety of the lunar surface (59). However, none of the seismically detected natural impacts have been connected with specific known craters. Connecting these types of seismic impact observations with orbital imaging of new craters would represent a leap forward in studies of the current impact flux, impact-seismic effects, and understanding the interior of the Moon. In addition, improved observations and assessments of the small impactor flux will be critical for assessing impact hazards for human and robotic exploration on the Moon and Mars.

\section{Recommendations}

The ideas and issues discussed in this paper represent some of the most pressing of modern lunar science. Understanding the chronology of the Moon is required to further our understanding of the evolution of both the Moon itself and the Earth-Moon system, and to anchor the chronologies of the other bodies in the inner solar system. Addressing the uncertainties in the recent flux, which represents a vital component of this chronological understanding, requires a multidisciplinary approach that treats the entire system rather than individual bodies alone. It should combine laboratory studies of multiple kinds of planetary samples, including meteorites that reveal clues about recent impacts on their parent bodies, with new approaches to the statistical treatment of impact cratering and new dynamical simulations of events affecting the Earth-Moon system. Critically, new returned samples are required; as outlined above, those samples must have known provenance and should include a variety of compositions, ages, and source regions. In addition, further refinement of in situ sample dating techniques $(68,69)$ and missions to return those samples (70) must be undertaken. Success will be achieved by employing a diverse workforce that draws on multiple types of expertise and experience, specialties, and resources to create an integrated end product that has more scientific influence than the sum of its individual parts (71).

New observations and analysis of craters on the Moon and other bodies, including Earth, is imperative. Though LRO continues to provide a wealth of new lunar observations, and the MESSENGER mission (72) brought studies of Mercury's surface into the modern era, specific types of observations are needed to further advance the field. New observations of Mercury's craters are needed to reveal how they differ in physical properties from lunar craters; this is required for robust interpretation of the statistical cratering record on Mercury. At the Moon, highresolution repeat imaging with long baselines allows detection of craters forming right now. Continued Earth-based investigations of micrometeorites and young craters and observations of lunar impact flashes, coupled with new work to quantify the small end of the impactor distribution 
represented by near-Earth objects (NEOs), will provide a critical direct observation of the current flux. Together, all of these observations will provide constraints for new dynamical models that will ultimately yield predictions and testable hypotheses to help unravel the evolution of the inner solar system as a whole.

\section{References}

1) Wood (2019) Geosci 9(1), 5. 2) Shoemaker (1962) Phys \& Astro of the Moon, Acad. Press, London, UK, pp. 283-359. 3) McEwen et al (1993) JGR 98, 17207-17231. 4) Neukum \& Ivanov (1994) in Hazards due to Comets and Asteroids, Univ of AZ Press, p. 359. 5) Marchi et al. (2009) $A J, 137,49361.6)$ Marchi et al. (2012) EPSL, 325-326, 27-38. 7) Morbidelli et al. (2012) EPSL 355-356, 144-151. 8) Robbins (2014) EPSL, 403, 188-198. 9) Kirchoff et al. (2013) Icarus 225, 325-341. 10) Kirchoff et al (2020) Suggestion that recent $(\leq 3 \mathrm{Ga})$ flux of $\mathrm{km}$ and larger impactors... Icarus (submitted). 11) Krogh et al (1993) Nature 366, 731-733. 12) Costello et al (2020) JGR: Planets, 125(3), e2019JE006172. 13) Ernst et al (2018) JGR: Planets, 123(10), 26282646. 14) Farrell et al (2019), GRL, 46(15), 8680-8688. 15) www.lpi.usra.edu/decadal/leag/ SCEMSummaryPieters.pdf. 16) www.lpi.usra.edu/leag/roadmap/. 17) Mazrouei et al (2019) Science, 363, 253-257. 18) Schmitz et al (2003) Science, 300(5621), 961-964. 19) Earth Impact Database (2011) http://www.unb.ca/passc/ImpactDatabase/. 20) Greenwood et al (2007) EPSL 262(1-2), 204-213. 21) Heck et al (2010) GCA, 74(2), 497-509. 22) Cronholm \& Schmitz (2010) Icarus, 208(1), 36-48. 23) Schmitz et al (2019b) Sci Adv, 5(9), eaax4184. 24) Heck et al (2017) Nature Astro., 1(2), 1-6. 25) Heck et al (2016) GCA, 177, 120-129. 26) Martin et al (2018) MAPS, 53(12), 2541-2557. 27) Schmitz et al (2019b) EPSL, 522, 234-243. 28) Caplan et al. (2019) 50th LPSC 2068.pdf. 29) Schmitz et al (2017) Geology, 45(9), 807-810. 30) Koeberl \& Ivanov (2019) MAPS, 1-13. 31) Keller et al (2019) $P N A S$, doi:10.1073/pnas.1804350116. 32) Zellner et al (2009) GCA, 73, 4590-4597. 33) Wasson \& Wang (1991) Meteoritics, 26, 161-167. 34) Bogard (1995) Meteoritics, 30, 244. 35) Righter et al (2015) MAPS, 50, 1790-1819. 36) Dykhuis et al (2014) Icarus, 243, 111-128. 37) Vokrouhlický et al (2017) $A J, 153,172-195$. 38) Glass \& Simonson (2013) Distal Impact Ejecta Layers, Springer. 39) Neukum et al (2001) Space Sci. Rev., 96, 5586. 40) Baldwin (1985) Icarus 61(1), 63-91. 41) Hiesinger et al. (2012) JGR, 117, E00H10. 42) Morota et al. (2009) MAPS, 44(8), 1115-1120. 43) Williams et al (2014) Icarus, 235(C), 23-36. 44) Strom \& Fielder (1968) Nature 217, 611. 45) Hartmann (1968), CoLPL, 145-156. 46) Williams et al (2018a) JGR, 123(9), 2380-262. 47) Williams et al. (2018b) in Dynamic Mars, 363-386, Soare et al., eds, Elsevier. 48) Schmitt et al (2017) Icarus, 298, 2-33. 49) Huang et al. (2019) 50th LPSC, 3010.pdf. 50) Lucchitta (1977) Icarus 30, 80-96. 51) Joy et al. (2012) Science, 336, 14261429. 52) Zellner \& Delano (2015) GCA, 161, 203-218. 53) Grier et al (2001) JGR, 106(E12), 32847-32862. 54) Bandfield et al (2011) JGR, 116, E00H02. 55) Ghent et al (2014) Geology, 42, 1059-1062. 56) Bandfield et al (2014) Icarus, 231, 221-231. 57) Powell et al (2019) 50th LPSC, 2238.pdf. 58) Suggs et al (2014) Icarus, 238, 23-36. 59) Oberst et al (2012) Planet. Space Sci., 74, 179-193.60) Speyerer et al (2016) Nature, 538, 215-218. 61) Speyerer et al (2020) 51st LPSC, 2369.pdf. 62) Daubar (2013) Icarus, 225(1), 506-16. 63) Rochette et al (2008) PNAS, 105, 1820618211. 64) Duennebier (1976) Science, 192, 1000-1002. 65) Oberst \& Nakamura (1991) Icarus $91,315-325.66)$ Longnonne et al (2009) JGR, 114, E12. 67) Gudkova et al (2014) Solar Sys. Res., 48, 11-21. 68) Anderson et al (2019) 50th LPSC, 3277.pdf. 69) Cohen et al (2014) Geostan Geoanal Res, 38. 70) Lawrence et al (2019) 50th LPSC, 1110.pdf. 71) Balakrishnan et al. (2011) https://dl.acm.org/doi/10.1145/1958824.1958905. 72) Solomon et al. (2007), Space Sci Rev, 131, 3-39. 